\title{
D-Egg: A next-generation optical module for IceCube
}

\author{
Yuya Makino $^{1, *}$ for the IceCube-Gen2 Collaboration \\ ${ }^{1}$ Department of Physics and Institute for Global Prominent Research, Chiba University, Chiba, 263- \\ 8522, Japan
}

\begin{abstract}
The Dual optical sensors in an Ellipsoid Glass for Gen2 (D$\mathrm{Egg}$ ) is one of the optical modules being developed for the IceCube-upgrade and the IceCube-Gen2 projects. The D-Egg has two 8-inch high-quantumefficiency PMTs facing downwards and upwards, which are coupled to a highly ultraviolet-transparent glass vessel to detect Cherenkov photons in nearly all directions. In this paper, expected performances of the D-Egg are discussed.
\end{abstract}

\section{Introduction}

The IceCube Neutrino Observatory [1] is a cubic kilometer Cherenkov detector in the deep ice at the South Pole. IceCube is planning to extend its neutrino detection capability in both higher and lower energies by installing additional photon sensors, which are usually called optical modules, around the current IceCube array as well as inside the DeepCore array.

The next-generation optical module for such detector extensions is expected to have improved detection efficiency for Cherenkov photons over the current IceCube Digital Optical Module (DOM). It is also anticipated that additional information possibly provided by new sensors, such as positions of the Cherenkov photons at each optical sensor, will lead to advances in event reconstructions. Furthermore, satisfying the requirements above, the module should have a smaller diameter to reduce the drilling cost.

\section{D-Egg}

The Dual optical sensors in an Ellipsoid Glass for Gen2 (D-Egg) [2] is a new optical module developed for the IceCube-upgrade and the IceCube-Gen2 projects [3]. $250 \mathrm{D}$-Eggs are planned to be deployed in the 2022-23 season as a part of the IceCube-upgrade array.

Figure 1(a) shows the picture of the prototype D-Egg module. A whole D-Egg module is $53 \mathrm{~cm}$ tall and $30 \mathrm{~cm}$ in diameter, which is $10 \%$ smaller than that of the DOM. Each D-Egg module stores two 8-inch high quantum-efficiency photomultiplier tubes (hereafter PMT; Hamamatsu Photonics R5912-100-20) facing upward and downward in an ellipsoid glass sphere (Okamoto Glass). The front-end electronics board inside the module reads the signals from both PMTs with $250 \mathrm{MHz}$ sampling and 14-bit resolution without dead-time. The PMTs are covered by Finemet cones for the magnetic shielding, and are coupled to the glass sphere with optical elastomers (Shinetsu Silicone) [1].

Since the intensity of Cherenkov photons increases at shorter wavelengths, improving UV-transparencies of the glass sphere and the optical elastomer is key to obtain a better

\footnotetext{
*e-mail: makino@hepburn.s.chiba-u.ac.jp
} 


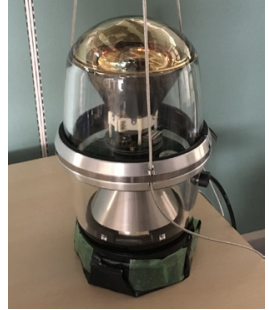

(a)

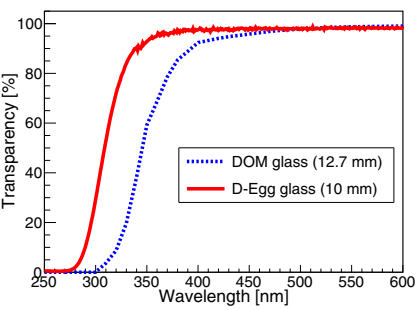

(b)

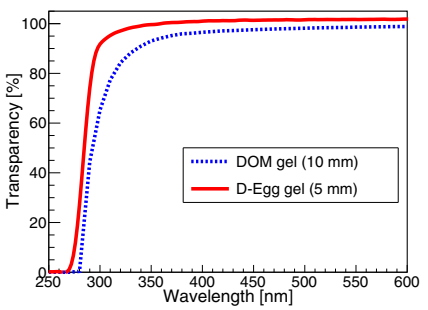

(c)

Figure 1. a) A prototype D-Egg module. b,c)Transparencies of the glasses and the optical elastomers. The solid-red and the dashed-blue lines represent those of the D-Egg and DOM with the corresponding thicknesses at the bottom, respectively.

detection efficiency. Figures 1(b) and 1(c) show the transparencies of the glass and the optical elastomer of the D-Egg and DOM. Compared to the DOM, the thicknesses of the glass and the elastomer for the D-Egg are reduced to $10 \mathrm{~mm}$ and $5 \mathrm{~mm}$, respectively, at the top (bottom) of the module. The particular improvement of UV-transmittance in the glass has been achieved by reducing the iron component to $0.006 \%$ by weight. Mechanical strength of the module sphere has been proven in both simulation studies and high pressure tests [2]. Transparencies of the glass and the optical elastomer for D-Egg are $75 \%$ and $95 \%$ at $320 \mathrm{~nm}$, whereas those of DOM are $10 \%$ and $85 \%$.

Although the effective area of a PMT in a D-Egg is $25 \%$ smaller than that of a DOM, one can expect a comparable or even better detection efficiency of Cherenkov photons in all directions by introducing a dual high-QE PMT system and the UV-transparent glass sphere and optical elastomers. In the following sections, we introduce the measured performances of the prototype half D-Egg modules (hereafter, "D-Egg" for simplicity). In Sec. 3, we discuss the wavelength and the angular dependences of the detection efficiency. In Sec. 4, we evaluate the expected effective area of a complete D-Egg module based on the lab measurements.

\section{Detection efficiency of prototype D-Eggs}

\subsection{Wavelength dependence}

Figure 2(a) shows the setup of the absolute detection efficiency measurement. A pulsed beam from the light emitting diode (LED) flasher splits into two at a neutral density (ND) filter; one goes to the pre-calibrated 1-inch PMT as a reference, and the other goes to a target D-Egg. The transmission to reflection ratio of photon intensities at the ND filter has been determined prior to the measurement. In such a way, we can calculate the number of photons at the target D-Egg. The expected number of photons is then compared with the measured number of photo-electrons to calculate the detection efficiency. The LED flasher has four different LEDs with wavelengths of $315 \mathrm{~nm}, 340 \mathrm{~nm}, 365 \mathrm{~nm}$, and $405 \mathrm{~nm}$.

As shown in Fig. 2(b), we confirmed high detection efficiency, $25 \%$ and $35 \%$ at $340 \mathrm{~nm}$ and $405 \mathrm{~nm}$, respectively. Observed data points are mostly consistent with the expected performances calculated from the data sheets of each component within the current estimation of the systematic uncertainties. The systematic error of the measurement mainly comes from the fraction of the reflection component at the ND filter.

\subsection{Angular dependence}

To measure the angular dependence of the detection efficiency, we have developed a twodimensional scan system as shown in Fig. 3(a). The system scans a PMT or D-Egg with 


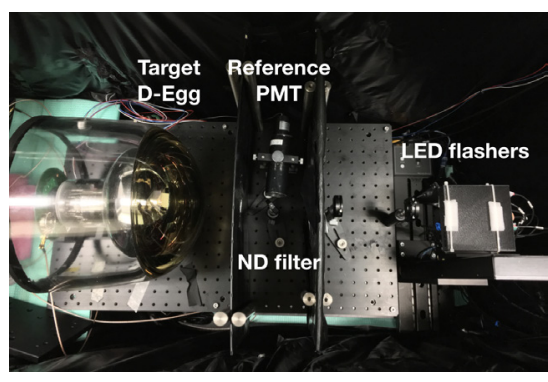

(a)

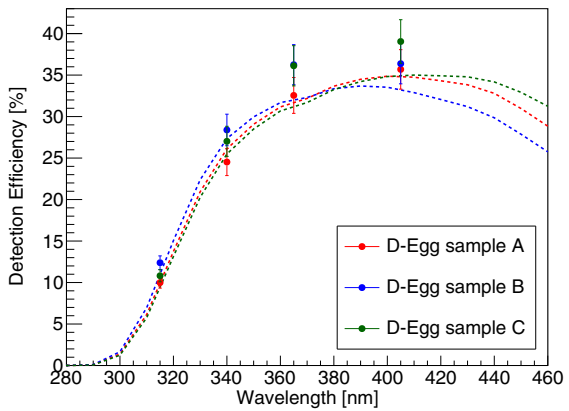

(b)

Figure 2. a) The setup of the absolute detection efficiency measurement. b) Wavelength dependence of the detection efficiency of three prototype D-Eggs. Points and dashed lines represent the observed results and the expectations calculated from the data sheet of each component, respectively. The results include the reflection at the glass surface.

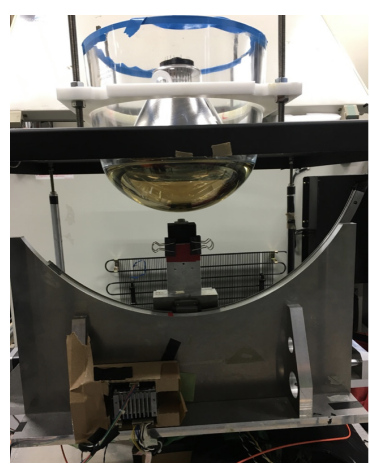

(a)

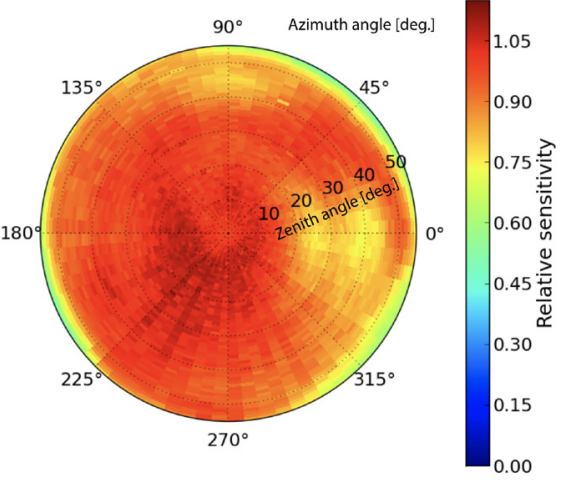

(b)

Figure 3. a) Two-dimensional scan system. The strength of the magnetic field is adjusted to match the one at the South Pole. b) Measured angular dependence of the sensitivity of a D-Egg. Sensitivities are normalized to the average value of the center region. The beam can point at the first stage of the dynode of a PMT when the azimuth and the zenith angle are around $0^{\circ}$ and $30^{\circ}$, respectively.

sampling step of $1^{\circ}$ and $5^{\circ}$ for zenith and azimuth angles, respectively. The light source of the system is a $400 \mathrm{~nm}$ picosecond laser (Hamamatsu C10196). The beam-spot size at the D-Egg or PMT is about $2 \mathrm{~mm}$ after the transportation of the light from the laser to the scan system through the optical fiber. The observed number of photo electrons is typically 20 at the center of D-Egg.

Figure 3(b) shows the angular dependence of the detection efficiency of a D-Egg. Although the observed angular dependence is uniform over the angles, some D-Eggs show a local minimum, which originates from the first dynode structure of the 8-inch PMT. Nonuniformity arise from the dynode structure strongly depends on the alignment between the PMT glass sphere and the dynode structure. 


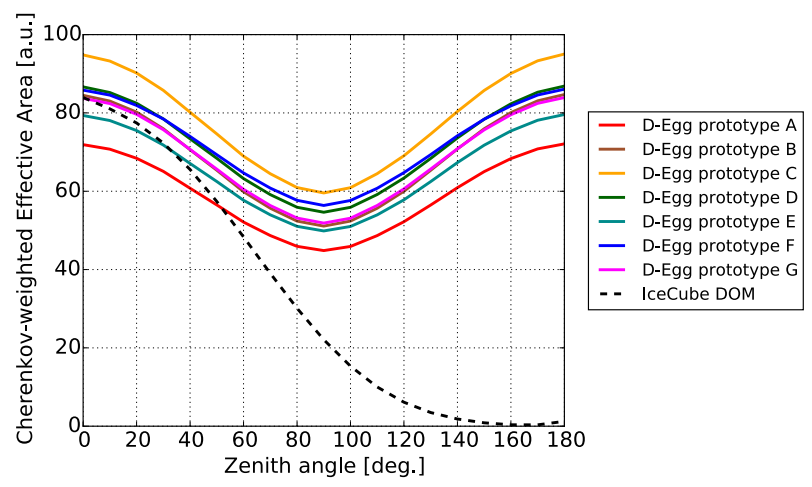

Figure 4. Cherenkov-spectrum-weighted effective area of DOM (dashed line) and D-Eggs (solid lines). Observed differences between D-Eggs are explained by the individual dependence of the PMT responses.

\section{Effective area of D-Egg module}

To evaluate the expected performance of the D-Egg in the glacier, we performed a study using a Monte Carlo simulator, DOMINANT [4], based on Geant4 [5]. In the simulation, a plane of photons hit the D-Egg in all directions. After the transportation of photons from the glass surface to the cathode of a PMT, photons are converted to photo electrons based on the measured responses of the PMTs, such as the quantum efficiency and the angular dependence, used for the corresponding D-Eggs. The obtained effective areas for each wavelength are then weighted according to the Cherenkov spectrum.

Figure 4 shows the Cherenkov-spectrum-weighted effective area of a DOM and the expected performances of D-Eggs. D-Eggs show high sensitivities over the zenith angle due to the dual-PMT system. The effective area of a D-Egg for the horizontal direction is $75 \%$ of that for the vertical. Overall, integrating the effective area along the zenith angles, D-Egg shows the performance of the Cherenkov-photon detection to be twice as good as that of DOM.

\section{Conclusion}

We are developing a new optical module for the next generation in-ice neutrino detector. We evaluated the wavelength and the angular dependences of the prototype D-Egg modules. The obtained results, $35 \%$ of the detection efficiency at $405 \mathrm{~nm}$ for instance, are satisfactory. The simulation study based on the laboratory measurements implies that the Cherenkov-photon detection performance of D-Egg is twice as good as that of the current standard DOMs used in IceCube.

\section{References}

[1] M.G. Aartsenet et. al, Journal of Instrumentation, 12, (2017).

[2] The IceCube-Gen2 Collaboration, and S. Shimizu, PoS(ICRC2017) 934.

[3] IceCube-Gen2 Collaboration, arXiv:1412.5106

[4] Homepage: http://www.ppl.phys.chiba-u.jp/research/IceCube/DetectorSim/DOMINANT/index.html.

[5] J. Allison et. al, Nuclear Instruments and Methods in Physics Research A 835, (2016). 